\title{
COMPACTIFICATIONS OF COUNTABLE-DIMENSIONAL AND STRONGLY COUNTABLE-DIMENSIONAL SPACES
}

\author{
RYSZARD ENGELKING AND ROMAN POL
}

(Communicated by Dennis Burke)

\begin{abstract}
Simple proofs of theorems on existence of compactifications of countable-dimensional and strongly countable-dimensional spaces are given.
\end{abstract}

In this note we present simple proofs of the following two theorems (for the terminology see [2 and 3]):

THEOREM 1. Every countable-dimensional completely metrizable separable space has a countable-dimensional metrizable compactification.

THEOREM 2. Every strongly countable-dimensional completely metrizable separable space has a strongly countable-dimensional metrizable compactification.

Let us recall that a completely regular space $X$ is (strongly) countable-dimensional if $X$ can be represented as a union of a sequence $X_{1}, X_{2}, \ldots$ of (closed) subspaces with covering dimension $\operatorname{dim} X_{i}<\infty$ (see [2] and [3]).

Theorem 1 was announced by Hurewicz in [5] and proved by Lelek in [8]. Theorem 2 was established by Schurle in [9].

Theorem 1 follows immediately from the Lemma we state below. The original proof of the Lemma sketched in [8] was based on the technique of Kuratowski's $\kappa$-mappings to infinite polyhedra; $\mathrm{cf}$. [7, §28, IX]; a short and elegant proof can be found in [4], it uses, however, some deep facts from infinite-dimensional topology. We give here an elementary proof.

LEMMA. For every completely metrizable separable space $X$ there exists an embedding $h: X \rightarrow Z$ into a compact metrizable space $Z$ such that $Z \backslash h(X)$ is a countable union of finite-dimensional compact spaces.

PrOOF. One can consider $X$ as a subspace of a compact metrizable space $Y$, and $X$ is the intersection of countably many open subsets $U_{1} \supset U_{2} \supset \cdots$ of $Y$ (see [2, Theorem 4.3.24]). Let $\rho$ be an arbitrary metric on the space $Y$ that is bounded by 1 . Since $\rho$ is totally bounded, for $i=1,2, \ldots$ the set $U_{i}$ can be represented as the union of finitely many open sets $U_{i j} \subset Y$, where $j \in J_{i}$, with diameter less than $1 / i$; let $f_{i j}: Y \rightarrow I$ be the continuous function defined by $f_{i j}(y)=\rho\left(y, Y \backslash U_{i j}\right)$ for $i=1,2, \ldots$ and $j \in J_{i}$. Denote by $f$ the diagonal of the functions $f_{i j}$; clearly, $f: Y \rightarrow I^{\aleph_{0}}$ and the restriction $h=f \mid X$ separates points and closed sets, and thus is an embedding of $X$ into $Z=\overline{f(X)}$. As one easily sees, $Y \backslash X=f^{-1}\left(K_{\omega}\right)$, where $K_{\omega}$ is the subspace of $I^{\aleph_{0}}$ consisting of all points that have only finitely

Received by the editors July 17, 1987 and, in revised form, November 16, 1987.

1980 Mathematics Subject Classification (1985 Revision). Primary 54F45. 
many nonzero coordinates; obviously, $K_{\omega}$ is the union of countably many finitedimensional cubes. Now, $Z \backslash h(X) \subset K_{\omega}$, and being an $F_{\sigma}$-set in $K_{\omega}$ is a countable union of finite-dimensional compact spaces.

In our proof of Theorem 2 we use a very special case of the separation theorem for Borel sets (see [7, §30, VII]), viz. the fact that for every pair $A, B$ of disjoint $G_{\delta}$-sets in a metrizable space $M$ there exists a set $C$ which is both an $F_{\sigma}$ and a $G_{\delta}$-set such that $A \subset C \subset M \backslash B$. This can be easily verified: if $A=\bigcap_{i=1}^{\infty} G_{i}$ and $B=\bigcap_{i=1}^{\infty} H_{i}$, where the sets $M=G_{1} \supset G_{2} \supset \cdots$ and $M=H_{1} \supset H_{2} \supset \cdots$ are open in $M$, the $F_{\sigma}$-set $C=\bigcup_{i=1}^{\infty}\left(G_{i} \backslash H_{i}\right)$ has the required properties, since $M \backslash C=\bigcup_{i=1}^{\infty}\left(H_{i} \backslash G_{i+1}\right)$ is an $F_{\sigma}$-set, too.

PROOF OF THEOREM 2. Consider a strongly countable-dimensional completely metrizable space $X$; let $X=\bigcup_{i=1}^{\infty} F_{i}$, where $F_{i}$ are finite-dimensional closed subsets of $X$. By a theorem of Hurewicz [6]; for a proof, see [7, §45, VII]), there exists a metrizable compactification $M$ of the space $X$ such that for $i=1,2, \ldots$ we have $\operatorname{dim} \bar{F}_{i}=\operatorname{dim} F_{i}$, where the closure is taken in $M$. The sets $A=X$ and $B=M \backslash \bigcup_{i=1}^{\infty} \bar{F}_{i}$ are disjoint $G_{\delta}$-sets in $M$, so that there exists a set $Y$ which is both an $F_{\sigma}$ and a $G_{\delta}$-set such that $X \subset Y \subset \bigcup_{i=1}^{\infty} \bar{F}_{i}$. The space $Y$ is a countable union of finite-dimensional compact spaces; it is also completely metrizable. so that by the Lemma it has a metrizable compactification $Z$ obtained by adjoining to $Y$ countably many finite-dimensional compact spaces. Obviously, $Z$ is a strongly countable-dimensional compactification of the space $X$.

To conclude, let us observe that our proof of the Lemma yields a more general result:

PROPOSITION 1. For every completely regular space $X$ that has the following property:

(*) $\quad X$ is the intersection of countably many open subsets $U_{1} \supset U_{2} \supset$ .. of a compact space $Y$ and for $i=1,2, \ldots$ the set $U_{i}$ can be represented as the union of a point-finite family $\left\{U_{i s}\right\}_{s \in S_{i}}$ of functionally open subsets of $Y$ in such a way that for every pair $x_{1}, x_{2}$ of distinct points of $X$ there exists an $i$ and an $s \in S_{i}$ such that the set $U_{i s}$ contains exactly one of the points $x_{1}, x_{2}$,

there exists an embedding $h: X \rightarrow Z$ into a compact space $Z$ such that $Z \backslash h(X)$ is a countable union of finite-dimensional compact spaces.

ProOF. Let $f_{i s}: Y \rightarrow I$ be a continuous function such that $f_{i s}^{-1}(0)=Y \backslash U_{i s}$ for $i=1,2, \ldots$ and $s \in S_{i}$. The diagonal $f$ of the functions $f_{i s}$ is a continuous mapping of $Y$ into a Tychonoff cube $I^{m}$, the restriction $h=f \mid X$ is a one-to-one mapping, and $Y \backslash X=f^{-1}(K)$, where $K$ is the subspace of $I^{m}$ consisting of all points that have only finitely many nonzero coordinates. Since $h$ is the restriction of the perfect mapping $f$ to the set $X=f^{-1}\left(I^{m} \backslash K\right)$, it is perfect itself (sce [2. Proposition 3.7.4]), and being one-to-one is an embedding of $X$ into $Z=\overline{f(X)}$. The remainder $Z \backslash h(X)$ is an $F_{\sigma}$-set in $Z$ (see [2, Theorem 3.9.1]) and is contained in $K$, so that to conclude the proof it suffices to show that $K$ is the union of countably many finite-dimensional compact spaces.

Clearly, $K=\bigcup_{n=0}^{\infty} K_{n}$, where $K_{n}$ consists of all points in $K$ that have at most $n$ nonzero coordinates. The subspaces $K_{n}$ being compact, it suffices to show that 
$\operatorname{dim} K_{n} \leq n$ for $n=0,1, \ldots$ This is done by induction. Since $K_{0}$ consists of one point, $\operatorname{dim} K_{0}=0$. Assume that $\operatorname{dim} K_{n} \leq n$. One easily checks that each point in $K_{n+1} \backslash K_{n}$ has a neighborhood in $K_{n+1}$ homeomorphic to the cube $(a, 1]^{n+1}$, where $0<a<1$; thus, for each closed set $F \subset K_{n+1}$ disjoint from $K_{n}$ we have $\operatorname{dim} F \leq n+1$ by the sum theorem, so that $\operatorname{dim} K_{n+1} \leq n+1$ (see [2, Theorem 7.2.1 and Problem 7.4.17]).

One easily checks that the class of completely regular spaces that have property $(*)$ is hereditary with respect to closed subspaces and countably multiplicative. Since every completely metrizable space is for some $m \geq \aleph_{0}$ homeomorphic to a closed subspace of $[J(m)]^{\aleph_{0}}$, where $J(m)$ is the hedgehog space of spininess $m$ (see [2, Exercise 4.4B]), to show that all completely metrizable spaces have property $(*)$, it suffices to find an appropriate compact space containing $J(m)$. Such a space is, however, easily defined (cf. the proof of Theorem 14 in [1]): if $S$ is the set of cardinality $m$ used in the construction of $J(m)$, as described in [2, Example 4.1.5], then the quotient space $Y=(I \times \omega S) /(\{0\} \times \omega S)$, where $\omega S$ is the one-point compactification of the discrete space $S$, has all the required properties.

Thus, one obtains the following proposition that answers a question in [3] (the first part of Problem 5.4):

PROPOSITION 2. Every countable-dimensional completely metrizable space has a countable-dimensional compactification.

\section{REFERENCES}

1. A. V. Arhangel'skiř, Bicompacta and unions of countable families of metrizable spaces, Dokl. Akad. Nauk SSSR 232 (1977), 989-992; English transl., Soviet Math. Dokl. 18 (1977), 165-169.

2. R. Engelking, General topology, PWN, Warsaw, 1977.

3. R. Engelking and E. Pol, Countable-dimensional spaces, Dissertationes Math. 216 (1983).

4. D. J. Garity and R. M. Schori, Infinite-dimensional dimension theory, Topology Proc. 10 (1985), 59-74.

5. W. Hurewicz, Über unendlich-dimensionale Punktmengen, Proc. Akad. Amsterdam 31 (1928), 916-922.

6. —_ Über Einbettung separabler Räume in gleichdimensionale kompakte Räume, Monatsh. Math. Phys. 37 (1930), 199-208.

7. K. Kuratowski, Topology, vols. I, II, Academic Press, New York, 1966 and 1968.

8. A. Lelek, On the dimension of remainders in compact extensions, Dokl. Akad. Nauk SSSR 160 (1965), 534-537; English transl., Soviet Math. Dokl. 6 (1965), 136-140.

9. A. Schurle, Compactification of strongly countable-dimensional spaces, Trans. Amer. Math. Soc. 136 (1969), 25-32.

Department of MAThematics, UNiVERsity of WARSAW, 00-901 WarszaWA, PKiN IX P., POLAND 